\title{
Green's Function for the Quartic Oscillator
}

\author{
Robert L. Anderson \\ Department of Physics and Astronomy, University of Georgia, Athens, USA \\ Email: andersonr@hal.physast.uga.edu
}

Received 28 June 2016; accepted 22 August 2016; published 25 August 2016

Copyright () 2016 by author and Scientific Research Publishing Inc.

This work is licensed under the Creative Commons Attribution International License (CC BY).

http://creativecommons.org/licenses/by/4.0/

(c) () Open Access

\section{Abstract}

In this paper, a quantum mechanical Green's function $G_{q o}\left(y_{b}, t_{b} ; y_{a}, t_{a}\right)$ for the quartic oscillator is presented. This result is built upon two previous papers: first [1], detailing the linearization of the quartic oscillator (qo) to the harmonic oscillator (ho); second [2], the integration of the classical action function for the quartic oscillator. Here an equivalent form for the quartic oscillator action function $S_{q o}\left(y_{b}, t_{b} ; y_{a}, t_{a}\right)$ in terms of harmonic oscillator variables is derived in order to facilitate the derivation of the quartic oscillator Green's Function, namely in fixing its amplitude.

\section{Keywords}

Nonrelativistic, Quartic, Quantum, Mechanics, Green's, Oscillator

\section{Introduction}

Following Schiff [3], the quantum mechanical Green's function $G\left(z_{2}, t_{2} ; z_{1}, t_{1}\right)$

$$
\Psi\left(z_{2}, t_{2}\right)=\int_{-\infty}^{\infty} G\left(z_{2}, t_{2} ; z_{1}, t_{1}\right) \Psi\left(z_{1}, t_{1}\right) \mathrm{d} z_{1},
$$

implements the superposition principle satisfied by the wave function because it satisfies a linear partial differential equation, the Schrödinger equation. (Note $z_{a}=0$ is included because of the integration.) Equation (1.1) implies that $G$ also satisfies it, namely in quartic qo variables:

$$
\frac{\hbar}{-i} \frac{\partial}{\partial t_{b}} G\left(y_{b}, t_{b} ; y_{a}, t_{a}\right)=\left\{\frac{1}{2 m}\left(\frac{\hbar}{i} \frac{\partial}{\partial y_{b}}\right)^{2}+V\left(y_{b}\right)\right\} G\left(y_{b}, t_{b} ; y_{a}, t_{a}\right) \text {. }
$$

Here we show that the Dirac-Feynman [4] [5] form

$$
G\left(y_{b}, t_{b} ; y_{a}, t_{a}\right)=\frac{1}{A\left[t_{b}, t_{a}\right]} \exp \frac{i}{\hbar} S\left(y_{b}, t_{b} ; y_{a}, t_{a}\right),
$$


where $S\left(y_{b}, t_{b} ; y_{a}, t_{a}\right)$ is the classical action for the quartic oscillator (qo). Thus, we will show that only the classical paths are needed in (1.1) for the qo as is true for the free particle and harmonic oscillator. We do not address the question of why the other non-classical paths in Feynman's path integral formulation [6] do not register.

Part II summarizes the results needed from [1].

Part III begins from first principles and expresses the Action function in terms of harmonic oscillator (ho) variables $S_{q o}\left(x_{z}, \hat{t}_{b} ; x_{a}, \hat{t}_{a}\right)$ and then integrates it. We establish that it is equal to that given in qo variables in [2]. This is fundamental to obtaining the correct value of the amplitude $A_{q o}$ appearing in the Green's function.

In Part IV, we then address the missing piece for the Green's function, namely, the Amplitude $A_{q o}\left(\hat{t}_{b}-\hat{t}_{a}\right)$. To obtain the target goals, these results are recast in terms of the qo variables. The Green's Function is then fixed in the final paragraph of this section.

Part V outlines the extension of these results to the hierarchy of all even power potentials.

\section{Review of Linearization Map}

The linearization map [1] implements the correspondence between the solutions to Newton's equations of motion for the ho and qo,

$$
m \frac{\mathrm{d}^{2}}{\mathrm{~d}^{2} \hat{t}} x(\hat{t})=-k_{2} x(\hat{t}) \Leftrightarrow m \frac{\mathrm{d}^{2}}{\mathrm{~d}^{2} t} y(t)=-k_{4} y^{3}(t) .
$$

Note both systems are assumed to have the same mass $m$.

Specifically, the invertible linearization map to the quartic oscillator with mass $m$ and space coordinate $y$ is stated in two parts. First,

$$
y=\left(2 k_{2} / k_{4}\right)^{1 / 4} x /\left(x^{2}\right)^{1 / 4}
$$

or

$$
x=\left(k_{4} / 2 k_{2}\right)^{1 / 2}\left(y^{2}\right)^{1 / 2} y,
$$

where $y$ is the space coordinate of the quartic oscillator and we have used the representation $\operatorname{sgn}(x)=x /\left(x^{2}\right)^{1 / 2}$ and similarly for $\operatorname{sgn}(y)$. This implements the physical requirement that $\frac{1}{2} k_{2} x^{2}(\hat{t})=\frac{1}{4} k_{4} y^{4}(t)$ i.e. matching the potential energies at the two different times, coupled with matching of the signs of the space coordinates. One cycle of the qo corresponds to one cycle of the ho, of course the periods are different.

Second,

$$
\frac{\mathrm{d} t}{\mathrm{~d} \hat{t}}=1 / 2\left(2 k_{2} / k_{4}\right)^{1 / 4}\left(x^{2}(\hat{t})\right)^{-1 / 4}
$$

and

$$
\frac{\mathrm{d} \hat{t}}{\mathrm{~d} t}=\left(2 k_{2} / k_{4}\right)^{1 / 2}\left(y^{2}(t)\right)^{1 / 2}
$$

which results by requiring

$$
\mathrm{d} x(\hat{t}) / \mathrm{d} \hat{t}=\mathrm{d} y(t) / \mathrm{d} t .
$$

Given the matching of the potential energies, the matching of the velocities and the masses of the oscillators for all values of $k_{2}$ and $k_{4}$ implies physically matching the momentum at the two different times and the kinetic energies, i.e. $p_{\text {ho }}(\hat{t})=p_{q o}(t), E_{\text {ho }}=E_{q o}=E$.

Further we need

$$
\begin{aligned}
& x(\hat{t})=\left[x_{b} \sin \omega\left(\hat{t}-\hat{t}_{a}\right)+x_{a} \sin \omega\left(\hat{t}_{b}-\hat{t}\right)\right] / \sin \omega\left(\hat{t}_{b}-\hat{t}_{a}\right), \\
& \frac{\left(y^{2}(t)\right)^{1 / 2} y(t)}{\left(4 E / k_{4}\right)^{1 / 2}}=\sin \left(\theta-\theta_{o}\right)=\frac{x_{\text {ho }}(\hat{t})}{\left(2 E / k_{2}\right)^{1 / 2}} .
\end{aligned}
$$


Note: Our convention $t=0 \leftrightarrow \hat{t}=0$. The physical significance of $t_{0}$ will be found in Part IV.

Finally, and key to the interchangeability of the qo and ho variables needed here is the standard change of variables in differentiation given by the following: First, it follows from (2.2) that

$$
\frac{\partial}{\partial y}=\frac{\partial x}{\partial y} \frac{\partial}{\partial x}=2\left(\frac{k_{4}}{2 k_{2}}\right)^{1 / 4}\left(x^{2}\right)^{1 / 4} \frac{\partial}{\partial x} \text {. }
$$

Second, it follows from (2.3) that

$$
\frac{\partial}{\partial t}=\frac{\partial \hat{t}}{\partial t} \frac{\partial}{\partial \hat{t}}=2\left(\frac{k_{4}}{2 k_{2}}\right)^{1 / 4}\left(x^{2}\right)^{1 / 4} \frac{\partial}{\partial \hat{t}} .
$$

Note from (2.7) and (2.8) that

$$
\frac{\partial x}{\partial y}=\frac{\partial \hat{t}}{\partial t}
$$

\section{The qo Action in Terms of the ho Variables}

As stated in the Introduction, the object of this paragraph is to express the defining expression for the qo action in terms of the ho variables and integrate it.

We start with an expression of the qo action in qo variables and transform it to ho variables

$$
\begin{aligned}
& S_{q o}=\int_{t_{a}}^{t_{b}}\left(\frac{1}{2} m\left(\frac{\mathrm{d} y(t)}{\mathrm{d} t}\right)^{2}-\frac{1}{4} k_{4} y^{4}(t)\right) \mathrm{d} t \\
& =\int_{\hat{t}_{a}}^{\hat{t}_{b}}\left(\frac{1}{2} m\left(\frac{\mathrm{d} x(\hat{t})}{\mathrm{d} \hat{t}}\right)^{2}-\frac{1}{2} k_{2} x^{2}(\hat{t})\right) \underbrace{\frac{1}{2}\left(\frac{2 k_{2}}{k_{4}}\right)^{1 / 4}\left(x^{2}(\hat{t})\right)^{-1 / 4}}_{\mathrm{d} t} \mathrm{~d} \hat{t} .
\end{aligned}
$$

Employing $\frac{1}{2} m\left(\frac{\mathrm{d} x(\hat{t})}{\mathrm{d} \hat{t}}\right)^{2}+\frac{1}{2} k_{2}(x(\hat{t}))^{2}=E$, we obtain from (3.1)

$$
\begin{aligned}
S_{q o} & =\int_{\hat{t}_{a}}^{\hat{t}_{b}}\left(m\left(\frac{\mathrm{d} x(\hat{t})}{\mathrm{d} \hat{t}}\right)^{2}-E\right) \frac{1}{2}\left(\frac{2 k_{2}}{k_{4}}\right)^{1 / 4}\left(x^{2}(\hat{t})\right)^{-1 / 4} \mathrm{~d} \hat{t} \\
& =\int_{\hat{t}_{a}}^{\hat{t}_{b}} m \frac{\mathrm{d} x(\hat{t})}{\mathrm{d} \hat{t}} \mathrm{~d} x \frac{1}{2}\left(\frac{2 k_{2}}{k_{4}}\right)^{1 / 4}\left(x^{2}(\hat{t})\right)^{-1 / 4} \mathrm{~d} \hat{t}-E\left(t_{b}-t_{a}\right),
\end{aligned}
$$

where

$$
t_{b}-t_{a}=\int_{\hat{t}_{a}}^{\hat{t}_{b}} \frac{1}{2}\left(\frac{2 k_{2}}{k_{4}}\right)^{1 / 4}\left(x^{2}(\hat{t})\right)^{-1 / 4} \mathrm{~d} \hat{t} .
$$

Now

$$
x(\hat{t})=\left(\frac{2 E}{k_{2}}\right) \sin \left(\theta(\hat{t})-\theta_{o}\left(\hat{t}_{o}\right)\right)
$$

where $\theta(\hat{t})=\omega \hat{t}$ and $k_{2}=m \omega^{2}$.

Therefore

$$
\mathrm{d} x(\hat{t})=\left(\frac{2 E}{k_{2}}\right)^{1 / 2} \cos \left(\theta(\hat{t})-\theta_{o}\left(\hat{t}_{o}\right)\right) \underbrace{\mathrm{d} \theta(\hat{t})}_{\omega \mathrm{d} \hat{t}},
$$


and

$$
\frac{\mathrm{d} x(\hat{t})}{\mathrm{d} \hat{t}}=\left(\frac{2 E}{k_{2}}\right)^{1 / 2} \cos \left(\theta(\hat{t})-\theta\left(\hat{t}_{o}\right)\right) \omega .
$$

Continuing, we have for the first term in the final expression (3.2a)

$$
=\frac{2 E}{m} \int_{\hat{t}_{a}}^{\hat{t}_{1}} \frac{1}{2}\left(\frac{2 k_{2}}{k_{4}}\right)^{1 / 4}\left(\frac{2 E}{k_{2}}\right)^{-1 / 4} \cos ^{2}\left(\theta(\hat{t})-\theta\left(\hat{t}_{o}\right)\right)\left(\sin ^{2}\left(\theta(\hat{t})-\theta\left(\hat{t}_{o}\right)\right)\right)^{-1 / 4} \mathrm{~d} \hat{t} .
$$

Therefore

$$
S_{q o}=\left[2 E\left(\frac{2 E}{k_{2}}\right)^{-1 / 4}\right]\left[\frac{1}{2}\left(\frac{2 k_{2}}{k_{4}}\right)^{1 / 4}\right] \int_{\hat{t}_{a}}^{\hat{t}_{b}} \cos ^{2}\left(\theta(\hat{t})-\theta\left(\hat{t}_{o}\right)\right)\left(\sin ^{2}\left(\theta(\hat{t})-\theta\left(\hat{t}_{o}\right)\right)\right)^{-1 / 4} \mathrm{~d} \hat{t}-E\left(t_{b}-t_{a}\right) .
$$

Now paralleling the development in [2] we effect the integration by parts, where:

$$
\frac{\mathrm{d}}{\mathrm{d} \hat{t}}(f g)=\frac{\mathrm{d} f}{\mathrm{~d} \hat{t}} g+f \frac{\mathrm{d} g}{\mathrm{~d} \hat{t}}
$$

with $f=\left(\sin ^{2}\left(\theta(\hat{t})-\theta\left(\hat{t}_{o}\right)\right)\right)^{3 / 4}$ and $g=\frac{2}{3} \frac{\cos \left(\theta(\hat{t})-\theta\left(\hat{t}_{o}\right)\right)}{\sin \left(\theta(\hat{t})-\theta\left(\hat{t}_{o}\right)\right)}$.

Therefore,

$$
\begin{aligned}
& \int_{\hat{t}_{a}}^{\hat{t}_{b}} \cos ^{2}\left(\theta(\hat{t})-\theta\left(\hat{t}_{o}\right)\right)\left(\sin ^{2}\left(\theta(\hat{t})-\theta\left(\hat{t}_{o}\right)\right)\right)^{-1 / 4} \mathrm{~d} \hat{t} \\
& =\frac{2}{3} \int_{\hat{t}_{a}}^{\hat{t}_{b}}\left(\sin ^{2}\left(\theta(\hat{t})-\theta\left(\hat{t}_{o}\right)\right)\right)^{-1 / 4} \mathrm{~d} \hat{t}+\left.\frac{2}{3} \frac{1}{\omega}\left(\sin ^{2}\left(\theta(\hat{t})-\theta\left(\hat{t}_{o}\right)\right)\right)^{3 / 4} \frac{\cos \left(\theta(\hat{t})-\theta\left(\hat{t}_{o}\right)\right)}{\sin \left(\theta(\hat{t})-\theta\left(\hat{t}_{o}\right)\right)}\right|_{\hat{t}_{a}} ^{\hat{t}_{b}}
\end{aligned}
$$

Hence,

$$
\begin{aligned}
& S_{q o}=\left[2 E\left(\frac{2 E}{k_{2}}\right)^{-1 / 4}\right]\left[\frac{1}{2}\left(\frac{2 k_{2}}{k_{4}}\right)^{1 / 4}\right]\left\{\frac{2}{3} \int_{\hat{t}_{a}}^{\hat{t}_{b}}\left(\sin ^{2}\left(\theta(\hat{t})-\theta\left(\hat{t}_{o}\right)\right)\right)^{-1 / 4} \mathrm{~d} \hat{t}\right. \\
& \left.+\left.\frac{2}{3} \frac{1}{\omega}\left(\sin ^{2}\left(\theta(\hat{t})-\theta\left(\hat{t}_{o}\right)\right)\right)^{3 / 4} \frac{\cos \left(\theta(\hat{t})-\theta\left(\hat{t}_{o}\right)\right)}{\sin \left(\theta(\hat{t})-\theta\left(\hat{t}_{o}\right)\right)}\right|_{\hat{t}_{a}} ^{\hat{t}_{b}}\right\}-E\left(t_{b}-t_{a}\right) \\
& =\frac{4 E}{3} \underbrace{\int_{\hat{t}_{a}}^{\hat{t}_{b}}\left(\frac{2 E}{k_{2}}\right)^{-1 / 4}\left[\frac{1}{2}\left(\frac{2 k_{2}}{k_{4}}\right)^{1 / 4}\right]\left(\sin ^{2}\left(\theta(\hat{t})-\theta\left(\hat{t}_{o}\right)\right)\right)^{-1 / 4} \mathrm{~d} \hat{t}}_{\left(t_{b}-t_{a}\right)} \\
& +\left.\frac{\left(\frac{2 k_{2}}{k_{4}}\right)^{1 / 4}\left(k_{2} m\right)^{1 / 2}}{3} \underbrace{\left(x^{2}(\hat{t})\right)^{3 / 4}}_{\left(\left(x^{2}(\hat{t})\right)^{1 / 2}\right)^{3 / 2}} \frac{\cos \left(\theta(\hat{t})-\theta\left(\hat{t}_{o}\right)\right)}{\sin \left(\theta(\hat{t})-\theta\left(\hat{t}_{o}\right)\right)}\right|_{\hat{t}_{a}} ^{\hat{t}_{b}}-E\left(t_{b}-t_{a}\right) .
\end{aligned}
$$

Finally,

$$
S_{q o}=\left.\frac{1}{3}\left(\frac{2 k_{2}}{k_{4}}\right)^{1 / 4}\left(k_{2} m\right)^{1 / 2}\left(\left(x^{2}(\hat{t})\right)^{1 / 2}\right)^{3 / 2} \frac{\cos \left(\theta(\hat{t})-\theta\left(\hat{t}_{o}\right)\right)}{\sin \left(\theta(\hat{t})-\theta\left(\hat{t}_{o}\right)\right)}\right|_{\hat{t}_{a}} ^{\hat{t}_{b}}+\frac{1}{3} E\left(t_{b}-t_{a}\right),
$$


where

$$
\left(t-t_{0}\right)=\int_{\hat{t}_{0}}^{\hat{t}} \frac{1}{2}\left(\frac{2 k_{2}}{k_{4}}\right)^{1 / 4}\left(x^{2}(\hat{t})\right)^{-1 / 4} \mathrm{~d} \hat{t},
$$

and

$$
E=\frac{1}{2} k_{2}\left[\left\{x_{b}^{2}+x_{a}^{2}-2 x_{b} x_{a} \cos \omega\left(\hat{t}_{b}-\hat{t}_{a}\right)\right\} / \sin ^{2} \omega\left(\hat{t}_{b}-\hat{t}_{a}\right)\right] .
$$

Now we verify that this is indeed $S_{q o}$; but expressed in ho variables. To do this we use the linearization tools described in Part II. In particular, from (2.6)

$$
\begin{aligned}
& \frac{\partial S_{q o}}{\partial y_{b}}=2\left(\frac{k_{4}}{2 k_{2}}\right)^{1 / 4}\left(x_{b}^{2}\right)^{1 / 2} \frac{\partial}{\partial x_{b}} S_{q o} \\
& =2\left(\frac{k_{4}}{2 k_{2}}\right)^{1 / 4}\left(x_{b}^{2}\right)^{1 / 2} \frac{1}{3}\left(\frac{2 k_{2}}{k_{4}}\right)^{1 / 4}\left(k^{2} m\right)^{1 / 2} \frac{3}{4}\left(x_{b}^{2}\right)^{1 / 4} 2 x_{b} \frac{\cos \omega\left(\hat{t}_{b}-\hat{t}_{o}\right)}{\sin \omega\left(\hat{t}_{b}-\hat{t}_{o}\right)} \\
& =\left(k_{2} m\right)^{1 / 2} x_{b} \frac{\cos \omega\left(\hat{t}_{b}-\hat{t}_{o}\right)}{\sin \omega\left(\hat{t}_{b}-\hat{t}_{o}\right)} \\
& =\left(\frac{m k_{4}}{2}\right)^{1 / 2}\left(y_{b}^{2}\right)^{1 / 2} y_{b} \frac{\cos \int_{t_{o}}^{t_{b}}\left(\frac{2 k_{4}}{m}\right)^{1 / 2}\left(y^{2}(t)\right)^{1 / 2} \mathrm{~d} t}{\sin \int_{t_{o}}^{t_{b}}\left(\frac{2 k_{4}}{m}\right)^{1 / 2}\left(y^{2}(t)\right)^{1 / 2} \mathrm{~d} t}=p_{b} .
\end{aligned}
$$

This checks with (4.3) in [2].

Next,

$$
\begin{aligned}
\frac{\partial S_{q o}}{\partial t_{b}} & =2\left(\frac{k_{4}}{2 k_{2}}\right)^{1 / 4}\left(x_{b}^{2}\right)^{1 / 4} \frac{1}{3}\left(\frac{2 k_{2}}{k_{4}}\right)^{1 / 4}\left(k^{2} m\right)^{1 / 2}\left(\left(x_{b}^{2}\right)^{1 / 2}\right)^{3 / 2}\left(\frac{-1}{\sin ^{2} \omega\left(\hat{t}_{b}-\hat{t}_{o}\right)}\right) \\
& =-\frac{4}{3} E+\frac{1}{3} E=-E .
\end{aligned}
$$

This checks with (4.3) in [2].

$$
\left(\text { Thus, } S \text { obeys the H-J equation } 0=\frac{\partial S}{\partial t_{b}}+H\left(y_{b}, \frac{\partial S}{\partial y_{b}}\right) .\right)
$$

Similarly

$$
\frac{\partial S_{q o}}{\partial x_{a}}=-p_{a} \quad \text { and } \quad \frac{\partial S_{q o}}{\partial t_{a}}=E .
$$

Using the results in Part II, (3.10) can be directly shown to be equal to the result (4.2) in [2], namely, it is equal to

$$
\begin{aligned}
& S_{q o}\left(y_{b}, t_{b} ; y_{a}, t_{a}\right) \\
& =\frac{1}{3}\left(\frac{m k_{4}}{2}\right)^{1 / 2}\left[\left(y_{a}^{2}\right)^{3 / 2} \frac{\cos \left(\theta_{b}-\theta_{o}\right)}{\sin \left(\theta_{b}-\theta_{o}\right)}-\left(y_{a}^{2}\right)^{3 / 2} \frac{\cos \left(\theta_{b}-\theta_{o}\right)}{\sin \left(\theta_{b}-\theta_{o}\right)}\right]+\frac{E}{3}\left(t_{b}-t_{a}\right), \\
& {\left[\left(y_{a}^{2}\right)^{3 / 2} \frac{\cos \int_{t_{o}}^{t_{b}}\left(\frac{2 k_{4}}{m}\right)^{1 / 2}\left(y^{2}\left(t^{\prime}\right)\right)^{1 / 2} \mathrm{~d} t^{\prime}}{\sin \int_{t_{o}}^{t_{b}}\left(\frac{2 k_{4}}{m}\right)^{1 / 2}\left(y^{2}\left(t^{\prime}\right)\right)^{1 / 2} \mathrm{~d} t^{\prime}}-\left(y_{a}^{2}\right)^{3 / 2} \frac{\cos \int_{t_{a}}^{t_{o}}\left(\frac{2 k_{4}}{m}\right)^{1 / 2}\left(y^{2}\left(t^{\prime}\right)\right)^{1 / 2} \mathrm{~d} t^{\prime}}{\sin \int_{t_{a}}^{t_{o}}\left(\frac{2 k_{4}}{m}\right)^{1 / 2}\left(y^{2}\left(t^{\prime}\right)\right)^{1 / 2} \mathrm{~d} t^{\prime}}\right]+\frac{E}{3}\left(t_{b}-t_{a}\right),}
\end{aligned}
$$


where

$$
E=\frac{1}{4} k_{4}\left[y_{b}^{4}+y_{a}^{4}-2\left(y_{b}^{2}\right)^{1 / 2} y_{b}\left(y_{a}^{2}\right)^{2} y_{a} \cos \left(\theta\left(t_{b}\right)-\theta\left(t_{a}\right)\right)\right] \text {, }
$$

and

$$
\theta(t)-\theta\left(t_{o}\right)=\int_{t_{o}}^{t}\left(\frac{2 k_{4}}{m}\right)^{1 / 2}\left(y^{2}\left(t^{\prime}\right)\right)^{1 / 2} \mathrm{~d} t^{\prime} .
$$

The significance of $t_{0}$ will be discussed in the latter paragraph of Part IV.

(It is important to correct some exponent typos in [2] (arXiv:1207.4376v2 [math-ph]). These corrections do not affect any of the results reported there or here. The correct exponents were used in arriving at the results reported. The minus sign on lhs of (2.8) should be a plus. The exponent in (2.9a) and (3.1) in [2] on the $\sin ^{2}$ terms should read $-1 / 4$. The terms involving $\left(\frac{2 k}{m}\right)$ in the integrands on pp 120-121 should all carry an exponent of $1 / 2$. The corresponding equations and pages should be corrected in the arXiv article. Sorry for any inconvenience, but again no errors in the final results!)

\section{Green's Function for the Quartic Oscillator}

Here, (assuming the Dirac-Feynman form of the Green's function) an amplitude $A_{q o}$ is identified such that the expression $A_{q o}^{-1} \exp \left(\frac{i}{\hbar} S_{q o}\right)$ satisfies the Schrödinger equation:

$$
\frac{\hbar}{-i} \frac{\partial}{\partial t_{b}} A_{q o}^{-1} \exp \left(\frac{i}{\hbar} S_{q o}\right)=\left\{\frac{1}{2 m}\left(\frac{\hbar}{i} \frac{\partial}{\partial y_{b}}\right)^{2}+\frac{1}{4} k_{4} y_{b}^{4}\right\} A_{q o}^{-1} \exp \left(\frac{i}{\hbar} S_{q o}\right),
$$

where $S_{q o}$ is given by (3.16)-(3.18) and $A$ is to be determined.

Thus we have on the lhs of (4.1)

$$
=\left\{\frac{\hbar}{i}\left(\frac{\partial A_{q o}}{\partial t_{b}} / A_{q o}^{2}\right)+\frac{\hbar}{-i} \frac{i}{\hbar}(-E) A_{q o}^{-1}\right\} \exp \frac{i}{\hbar}(S),
$$

where $\frac{\partial S_{q o}}{\partial t_{b}}=-E$ from (3.14).

And we have the rhs of (3.1)

$$
=\left\{\hbar / 2 m i \frac{\partial p_{b}}{\partial y_{b}}+\frac{1}{2} m p_{b}^{2}+V(y)\right\} A_{q o}^{-1} \exp \frac{i}{\hbar}(S),
$$

where $\frac{\partial S}{\partial y_{b}}=p_{b}$ from (3.13).

The 2nd term in the lhs of (4.1) is equal to the sum of the 2nd and 3rd terms in the rhs of (4.1) for our conservative system.

This leaves only the 1st term of the lhs of (4.1) and the 1st term in the rhs of (4.1). Equating their coefficients and cancelling common factors we obtain

$$
\frac{\partial A_{q o}}{\partial t_{b}} / A_{q o}=2 m \frac{\partial p_{b}}{\partial y_{b}} .
$$

Proceeding with the evaluation of (4.4), we have for the lhs

$$
\frac{1}{A_{q o}} 2\left(\frac{k_{4}}{2 k_{2}}\right)^{1 / 4}\left(x_{b}^{2}\right)^{1 / 4} \frac{\partial}{\partial \hat{t}_{b}} A_{q o},
$$


and for the rhs

$$
\frac{1}{2 m} 2\left(\frac{k_{4}}{2 k_{2}}\right)^{1 / 4}\left(x_{b}^{2}\right)^{1 / 4} \frac{\partial}{\partial \hat{x}_{b}}\left(k^{2} m\right)^{1 / 2} \frac{\cos \omega\left(\hat{t}_{b}-\hat{t}_{o}\right)}{\sin \omega\left(\hat{t}_{b}-\hat{t}_{o}\right)} .
$$

Therefore equating (4.5) to (4.6) and canceling the common factors including one given by (2.9), we have

$$
A_{q o} \frac{\partial}{\partial \hat{t}} A_{q o}=\frac{1}{2} \omega \frac{\cos \omega\left(\hat{t}_{b}-\hat{t}_{o}\right)}{\sin \omega\left(\hat{t}_{b}-\hat{t}_{o}\right)},
$$

or

$$
\frac{\partial}{\partial \hat{t}} \ln A_{q o}=\frac{1}{2} \frac{\partial}{\partial \hat{t}} \ln \alpha \sin \omega\left(\hat{t}_{b}-\hat{t}_{o}\right)
$$

or

$$
A_{q o}=\left(\alpha \sin \omega\left(\hat{t}_{b}-\hat{t}_{o}\right)\right)^{1 / 2},
$$

where $\alpha=$ constant.

Before completing our discussion of the amplitude, we start with the observation that there exists a $z_{1}=0$ at $t_{1}$. Now this $z_{1}=0$ corresponds to a $\hat{z}_{1}=0$ at $\hat{t}_{1}$ via (2.2). This implies via (2.6) that $\hat{t}_{0}=\hat{t}_{1}$.

Now set $\hat{t}_{1}$. This implies $t_{1}$ via the quadrature (3.11).

Now set $\hat{t}_{2}$ and this implies $t_{2}$ via another application of the quadrature (3.11).

$x_{2}=x\left(\hat{t}_{2}\right)$ implies $y_{2}=y\left(t_{2}\right)=z_{2}\left(t_{2}\right)$ via (2.2). Therefore returning to the amplitude, we have

$$
A_{q o}=\left(\alpha \sin \omega\left(\hat{t}_{2}-\hat{t}_{1}\right)\right)^{1 / 2} .
$$

Here using $A_{q o}$ in the limit, which is ( $\alpha \omega \varepsilon$ ), where $\varepsilon$ is the increment in ho time, we link up with the development in F-H [6] for any system for which $H=T+V$ on pp 76 - 78 and find

$$
\alpha=\frac{2 \pi i \hbar}{m \omega} \text {. }
$$

Equivalently from (3.18)

$$
A_{q o}=\left(\frac{2 \pi i \hbar}{m \omega}\right) \sin \left[\int_{t_{1}}^{t_{2}}\left(\frac{2 k_{4}}{m}\right)^{1 / 2}\left(y^{2}(t)\right)^{1 / 2} \mathrm{~d} t\right] .
$$

We turn to how do we use this structure of the Green's function to bring it to the form (1.1).

There are two quadratures necessary to fix the connection between the coordinates.

To obtain (1.1) we set $z_{2}=y_{b}, t_{2}=t_{b}, z_{1}=y_{a}, t_{1}=t_{a}$ and note that the integrations overall $z_{1}$ technically eliminates knowing the exact connection; except for $z_{1}=0_{1}$, between the integration variable $z_{1}$ and $t_{1}$ because $t_{1}$ is fixed and the integration is over all values of $z_{1}$, where $z_{1}$ appears in $E$ given by (3.12). Therefore with these substitutions understand the $G\left(y_{b}, t_{b}, y_{a}, t_{0}\right)$ in our Green's function (1.3) is given by (3.16)-(3.18) and the amplitude $A\left[t_{b}, t_{0}\right]$ is given by (4.8) and (4.10)-(4.11) where $\omega\left(\hat{t}_{b}-\hat{t}_{a}\right)$ equals the rhs of (3.18). Again, there are only two quadratures with this application fixed by $\left(y_{b}=z_{2}, t_{b}=t_{2}\right)$ and $\left(y_{a}=z_{1}=0, t_{a}=t_{1}\right)$.

\section{Extremal Mapping for $\left.\frac{1}{2 n} k_{2 n} y_{2 n}^{2 n}(t)\right|_{n>1}$ Hierarchy}

In this section we present a brief outline of the extension of these results to the hierarchy of attractive potentials given by even powers of the space coordinate [1].

Fundamental to this outline the mapping of the harmonic oscillator extremals onto the extremals of a each member of an hierarchy of attractive oscillators with coordinates $y_{2 n}(t) ; n=2,3,4, \cdots$ characterized by even positive power law potentials. (The case, $y \equiv y_{4}$ which is included in the hierarchy, has been the subject of the 
preceding paragraph.) In a straight forward manner the mappings in Part II and Part 1, generalize and yield the following relationships:

$$
\begin{gathered}
y_{2 n}=\left(n k_{2} / k_{2 n}\right)^{1 / 2 n} x\left(x^{2}\right)^{(1 / 2)(1-n) / n} \\
x=\left(k_{2 n} / n k_{2}\right)^{1 / 2} y_{2 n}\left(y_{2 n}^{2}\right)^{(n-1) / 2},
\end{gathered}
$$

which is the generalization of (2.1). The generalization of (2.2) is given by:

$$
\frac{\mathrm{d} t}{\mathrm{~d} \hat{t}}=n^{-(2 n-1) / 2 n}\left(k_{2} / k_{2 n}\right)^{1 / 2 n}\left(x^{2}(\hat{t})\right)^{-(n-1) / 2 n}(\hat{t}),
$$

and

$$
\frac{\mathrm{d} \hat{t}}{\mathrm{~d} t}=\sqrt{n}\left(k_{2 n} / k_{2}\right)^{1 / 2}\left(y_{2 n}^{2}\right)^{(n-1) / 2},
$$

and

$$
\hat{t}_{o}=t_{o} \text {. }
$$

These mappings take the space-time extremals of the linear oscillator with coordinates $(x, \hat{t})$ and map them onto the space-time extremals of the (2n)th oscillator with coordinates $\left(y_{2 n}, t\right)$.

With these mapping in hand, all of the analyses presented in Parts II - IV can then be extended to the members of the hierarchy including the analysis of the corresponding $A_{2 n}$. It will be tedious.

\section{Conclusions}

A quantum mechanical Green's function $G\left(y_{b}, t_{b} ; y_{a}, t_{a}\right)$ (1.3) for the quartic oscillator is presented where $S_{q o}\left(y_{b}, t_{b} ; y_{a}, t_{a}\right)$ is given by (3.16)-(3.18) and $A_{q o}\left(t_{b}, t_{a}\right)$ is given by (4.9)-(4.10) with $y_{a}=0, t_{a}=0$. See the end of Part V. This result is built upon two previous papers: first [1], detailing the linearization of the quartic oscillator (qo) to the harmonic oscillator (ho); second [2], the integration of the classical action function for the quartic oscillator. Here an equivalent form for the quartic oscillator action function $S_{q o}\left(y_{b}, t_{b} ; y_{a}, t_{a}\right)$ in terms of harmonic oscillator variables is given by (3.10)-(3.12) in order to facilitate the derivation of the quartic oscillator Green's Function amplitude. To implement (1.1) set $y_{b}=y_{2}, t_{o}=t_{z}, y_{a}=y_{1}, t_{a}=t_{1}$ in the above.

The linearization map originally given in [1] and described in parts in Part II and IV of this paper describes the difference between our approach and that of R.C. Santos, J. Santos and J.A.S. Lima [7] who first reported the possibility of linearization of the qo to the ho.

\section{Acknowledgements}

The author wishes to thank Professor Howard Lee for insightful discussions and his constant encouragement. The idea to emphasize the quartic oscillator was his.

Finally, the author wishes to acknowledge those who participated in a seminar organized by Robert Varley and David Edwards in AY 2006-2007 to study Feynman Path Integrals, and especially two students, Emily Pritchett and Justin Manning. The seminar provided the original motivation for exploring the extent of the connection between the linear oscillator and the Feynman's Path Integral Method. As an offshoot of this seminar, special thanks go to my Department of Mathematics colleague Robert Varley, who spent enumerable hours over a four year period of time following the seminar discussing this work with me. His comments, questions and posing of challenging related problems helped to clarify for me many aspects of this work.

\section{References}

[1] Anderson, R.L. (2010) An Invertible Linearization Map for the Quartic Oscillator. Journal of Mathematical Physics, 51, Article ID: 122904. http://dx.doi.org/10.1063/1.3527070

[2] Anderson, R.L. (2013) Integration of the Classical Action for the Quartic Oscillator in 1+1 Dimensions. Applied Mathematics, 4, 117-122. http://dx.doi.org/10.4236/am.2013.410A3014

[3] Schiff, L.I. (1955/1968) Quantum Mechanics. 3rd Edition, McGraw-Hill, New York, p. 306. 
[4] Dirac, P.A.M. (1958) The Principle of Quantum Mechanics. 4th Edition, Oxford at the Clarendon Press, p. 128.

[5] Brown, L.M. (Ed.) (2005) Feynman's Thesis-A New Approach to Quantum Theory. World Scientific, Singapore.

[6] Feynman, R.P. and Hibbs, A.R. (1965) Quantum Mechanics and Path Integrals. McGraw-Hill, New York.

[7] Santos, R.C., Santos, J. and Lima, J.A.S. (2006) Hamilton-Jacobi Approach for Power-Law Potentials. Brazilian Journal of Physics, 36, 1257-1261. http://dx.doi.org/10.1590/S0103-97332006000700024

\section{Submit or recommend next manuscript to SCIRP and we will provide best service for you:}

Accepting pre-submission inquiries through Email, Facebook, LinkedIn, Twitter, etc.

A wide selection of journals (inclusive of 9 subjects, more than 200 journals)

Providing 24-hour high-quality service

User-friendly online submission system

Fair and swift peer-review system

Efficient typesetting and proofreading procedure

Display of the result of downloads and visits, as well as the number of cited articles

Maximum dissemination of your research work

Submit your manuscript at: http://papersubmission.scirp.org/ 